\title{
Progress on the Traceability Conjecture for Oriented Graphs
}

\author{
Marietjie Frick $\|$ and Peter Katrenič $\|$ \\ ${ }^{1}$ University of South Africa, P.O. Box 392, UNISA, 0003 South Africa \\ frickm@unisa.ac.za \\ ${ }^{2}$ P.J. Šafárik University, Jesenná 5, 04154 Košice, Slovak Republic \\ peter.katrenic@upjs.sk
}

received April 2, 2008, revised August 11, 2008, accepted October 23, 2008.

A digraph is $k$-traceable if each of its induced subdigraphs of order $k$ is traceable. The Traceability Conjecture is that for $k \geq 2$ every $k$-traceable oriented graph of order at least $2 k-1$ is traceable. The conjecture has been proved for $k \leq 5$. We prove that it also holds for $k=6$.

Keywords: longest path, oriented graph, nontraceable, k-traceable, Traceability Conjecture, Path Partition Conjecture

\section{Introduction}

The set of vertices and the set of arcs of a digraph $D$ are denoted by $V(D)$ and $A(D)$, respectively, and the order of $D$ is denoted $n(D)$. A directed cycle (path, walk) in a digraph will simply be called a cycle (path, walk). A digraph is hamiltonian if it contains a cycle that visits every vertex, traceable if it contains a path that visits every vertex, walkable (or unilaterally connected) if it contains a walk that visits every vertex, and strong (or strongly connected) if it has a closed walk that visits every vertex.

The maximum number of vertices on a path in a digraph $D$ is denoted by $\lambda(D)$. A digraph $D$ of order $n$ is $p$-deficient if $\lambda(D)=n-p$.

A maximal strong subdigraph of a digraph $D$ is called a strong component of $D$. We say that a strong component is trivial if has only one vertex.

If $v$ is a vertex of a digraph $D$, we denote the sets of out-neighbours and in-neighbours of $v$ by $N^{+}(v)$ and $N^{-}(v)$ and the cardinalities of these sets by $d^{+}(v)$ and $d^{-}(v)$, respectively. The minimum degree of $D, \delta(D)$, is defined as $\min _{v \in V(D)}\left(d^{+}(v)+d^{-}(v)\right)$.

If $D$ is a digraph and $X \subset V(D)$, then $\langle X\rangle$ denotes the subdigraph induced by $X$ in $D$.

A digraph of order $n$ is $k$-traceable for some $k \leq n$ if each of its induced subdigraphs of order $k$ is traceable. The main topic of this paper is the following conjecture, which was formulated by Morten Nielsen in 2006. It is stated in (5).

\footnotetext{
${ }^{\dagger}$ This material is based upon work supported by the National Research Foundation of S.A. under Grant number 2053752

¥The research of the author is partially supported by Slovak VEGA Grant 1/3004/06 and Slovak VVGS UPJŠ Grant 11/07-08 
Conjecture 1.1 (The Traceability Conjecture (TC)) For $k \geq 2$, every $k$-traceable oriented graph of order at least $2 k-1$ is traceable.

The Traceability Conjecture was inspired by the Path Partition Conjecture for 1-deficient Oriented Graphs (called the OPPC(1)), which is treated in (5) and (10). The OPPC(1) is an important special case of the Path Partition Conjecture for Digraphs (DPPC), which is treated in (2) and (4). The OPPC(1) may be stated as follows in terms of traceability (see (5)).

Conjecture 1.2 (OPPC(1)) Let $a$ and $b$ be integers with $1 \leq a \leq b$. If $D$ is a 1-deficient oriented graph of order $n=a+b+1$, then $D$ is not $(a+1)$-traceable or $D$ is not $(b+1)$-traceable.

The truth of the TC would obviously imply the truth of the OPPC(1). In particular, if the TC holds for $k=t$, it would follow that the OPPC(1) holds for $a=t-1$.

In the case of undirected graphs, it is an easy corollary of Dirac's degree condition for hamiltonicity that for $k \geq 2$ every $k$-traceable graph of order at least $2 k-1$ is hamiltonian. The same is not true for oriented graphs, though we do have the following result, which is proved in (5).

Theorem 1.3 For $k=2,3$ or 4 , every strong $k$-traceable oriented graph of order greater than $k$ is hamiltonian.

For $k \geq 5$ the situation changes dramatically. As shown in (5), for every $n \geq 5$ there exists a nonhamiltonian strong oriented graph of order $n$ that is $k$-traceable for every $k \in\{5, \ldots, n\}$. However, no counterexample to the TC has yet been found. In fact, we do not even know if there exists a $k$-traceable oriented graph of order bigger than $k+1$ that is nontraceable.

It is shown in (5) that the TC holds for $k \leq 5$. In this paper we prove that the TC also holds for $k=6$, i.e. every 6-traceable oriented graph of order at least 11 is traceable.

\section{Auxiliary Results}

First, we present some general properties of $k$-traceable oriented graphs. The following result concerning the minimum degree is proved in (5).

Lemma 2.1 If $k \geq 2$ and $D$ is a $k$-traceable oriented graph of order $n \geq k$, then $\delta(D) \geq n-k+1$.

Our next result concerns $k$-traceable oriented graphs that are nontraceable.

Lemma 2.2 Suppose $D$ is a $k$-traceable oriented graph of order $n>k$. If $D$ is nontraceable and $v$ is a vertex of $D$ with $d^{+}(v)=n-k+1$ then $\left\langle N^{+}(v)\right\rangle$ is nontraceable. Similarly, if $d^{-}(v)=n-k+1$, then $\left\langle N^{-}(v)\right\rangle$ is nontraceable.

Proof: Suppose $\left\langle N^{+}(v)\right\rangle$ has a hamiltonian path $x_{1} x_{2} \ldots x_{n-k+1}$. Then the graph $D-\left\{x_{1}, \ldots x_{n-k}\right\}$ has order $k$ and therefore has a hamiltonian path $P$. If $P$ contains the $\operatorname{arc} v x_{n-k+1}$, then the path obtained from $P$ by replacing the arc $v x_{n-k+1}$ with the path $v x_{1} x_{2} \ldots x_{n-k} x_{n-k+1}$ is a hamiltonian path of $D$. If $P$ does not contain the arc $v x_{n-k+1}$, then $v$ is the end-vertex of $P$. In this case $P x_{1} x_{2} \ldots x_{n-k}$ is a hamiltonian path of $D$. The proof that $\left\langle N^{-}(v)\right\rangle$ is nontraceable if $d^{-}(v)=n-k+1$ is similar.

The following easy observation is proved in (5).

Lemma 2.3 If $D$ is an oriented graph of order $n$ that is $k$-traceable for some $k \in\{2, \ldots, n\}$, then $D$ is walkable. 
In view of Lemma 2.3 we shall be mainly concerned with walkable oriented graphs. The strong components of a digraph have an acyclic ordering, i.e. they may be labelled $D_{1}, \ldots, D_{h}$ such that if there is an arc from $D_{i}$ to $D_{j}$, then $i \leq j$ (cf. (1), p. 17). If $D$ is walkable then, for $i=1, \ldots, h-1$ there is at least one arc from $D_{i}$ to $D_{i+1}$, so in this case the acyclic ordering is unique. Throughout the paper we shall label the strong components of a walkable oriented graph in accordance with this unique acyclic ordering.

In the proof of our main result we shall consider oriented graphs that are strong and those that are not strong (but walkable) separately. The nonstrong case relies on the following three results concerning the strong components of $k$-traceable oriented graphs. The first is an obvious but useful result, proved in (5).

Lemma 2.4 If $P$ is a path in a digraph $D$, then the intersection of $P$ with any strong component of $D$ is either empty or a path.

The next result follows from Theorem 1.3 and Lemma 2.4 .

Lemma 2.5 Let $k \geq 5$ and suppose $D$ is a $k$-traceable oriented graph of order $n>k$. Then every nonhamiltonian nontrivial strong component of $D$ has order at least $n-k+5$.

Proof: Suppose $D$ has a nonhamiltonian nontrivial strong component $X$ of order at most $n-(k-4)$. Then $|V(X)| \geq 4$ and $|V(D) \backslash V(X)| \geq k-4$. If $|V(X)|=4$, then $|V(D) \backslash V(X)|=n-4 \geq k-3$. Theorem 1.3 implies that $X$ is not 3-traceable and, if $|V(X)|>4$, then $X$ is also not 4-traceable. In either case, we can choose an induced subdigraph $H$ of $D$ of order $k$ such that $\langle V(H) \cap V(X)\rangle$ is nontraceable. But then it follows from Lemma 2.4 that $H$ is nontraceable, contradicting our assumption that $D$ is $k$-traceable.

The following result, which is proved in (5), is very useful in the case of $k$-traceable oriented graphs of large enough order.

Lemma 2.6 Suppose $D$ is a $k$-traceable oriented graph of order at least $2 k-1, k \geq 2$. If $D$ is nontraceable, then $D$ has a nonhamiltonian nontrivial strong component.

For the proof of the strong case of our main result, we shall use the following theorem, proved in (3).

Theorem 2.7 (Chen and Manalastas) Every nontraceable strong digraph has independence number at least 3 .

We shall also use the following result on $k$-traceable strong oriented graphs, which appears as part of the proof of Theorem 3.5 in (5).

Lemma 2.8 Let $D$ be a k-traceable strong oriented graph and let $X=\left\{x_{1}, x_{2}, x_{3}\right\}$ be an independent set of vertices in D. Let

$$
A_{i}=V(D) \backslash\left\{X \cup N^{-}\left(x_{i}\right)\right\}, B_{i}=V(D) \backslash\left\{X \cup N^{+}\left(x_{i}\right)\right\} ; i=1,2,3 .
$$

Then $\left|A_{i}\right| \leq 3 k-12$ and $\left|B_{i}\right| \leq 3 k-12$ for $i=1,2,3$.

Proof: Let $i, j$ be any pair of distinct integers in $\{1,2,3\}$. If $\left|A_{i} \cap A_{j}\right| \geq k-3$, let $H$ be an induced subdigraph of $D$ whose vertex set consists of $x_{1}, x_{2}, x_{3}$ and $k-3$ vertices of $A_{i} \cap A_{j}$. Then $H$ has order $k$ and is nontraceable, since both $x_{i}$ and $x_{j}$ have no in-neighbours in $H$. This contradiction shows that $\left|A_{i} \cap A_{j}\right| \leq k-4$. Similarly, $\left|B_{i} \cap B_{j}\right| \leq k-4$. Now suppose $\left|A_{1} \cap B_{2}\right| \geq 2 k-7$. Then, since $\left|A_{1} \cap A_{3}\right| \leq k-4$, at most $k-4$ vertices of $A_{1} \cap B_{2}$ are in $A_{3}$, so at least $k-3$ vertices of 
$A_{1} \cap B_{2}$ are in $B_{3}$, but then $\left|B_{2} \cap B_{3}\right| \geq k-3$. This contradiction proves that $\left|A_{1} \cap B_{2}\right| \leq 2 k-8$. But $A_{1}=\left(A_{1} \cap A_{2}\right) \cup\left(A_{1} \cap B_{2}\right)$. Hence $\left|A_{1}\right| \leq(k-4)+2 k-8=3 k-12$. Similarly, $\left|B_{1}\right| \leq 3 k-12$.

Theorem 2.7 and Lemma 2.8 were used in (5) to prove the following theorem.

Theorem 2.9 For $k \geq 2$ every $k$-traceable strong oriented graph of order at least $6 k-20$ is traceable.

We now turn our attention to $k$-traceable oriented graphs of small order. Knowledge of their structure is actually important when considering the traceability of $k$-traceable oriented graphs of large order.

\section{Hypotraceable oriented graphs}

A digraph $D$ of order $n$ is called hypotraceable if $n \geq 3$ and $D$ is $(n-1)$-traceable but not $n$-traceable. Thus a hypotraceble digraph is nontraceable but the removal of any vertex leaves a traceable digraph. Our next result shows the importance of hypotraceable oriented graphs in connection with the TC.

Lemma 3.1 If $k>2$ and $D$ is a nontraceable, $k$-traceable oriented graph of order $n \geq k+1$, then $D$ has a hypotraceable induced subdigraph of order $h$ for some $k+1 \leq h \leq n$.

Proof: Suppose $n=k+r$. Then, for any set $S$ consisting of $r$ vertices of $D$, the oriented graph $D-S$ is traceable. If $D$ itself is not hypotraceable, then $D$ has a vertex $x_{1}$ such that $D-x_{1}$ is nontraceable. We repeat this procedure until we obtain a subset $\left\{x_{1}, \ldots, x_{t}\right\}$ in $D$ for some $t \leq r-1$ such that $D-\left\{x_{1}, \ldots, x_{t}\right\}$ is hypotraceable.

In view of Lemma 3.1 it is important to know the possible orders of hypotraceable oriented graphs. Grötschel, Thomassen and Wakabayashi constructed an infinite family of hypotraceable oriented graphs in (6). These graphs are obtained from hypohamiltonian digraphs. The smallest hypotraceable oriented graph constructed by applying the construction in (6) to hypohamiltonian digraphs constructed by Thomassen in (9) has order 12. If there does not exist a hypotraceable oriented graph of order less than 12, then it would follow immediately from Theorem 2.9 and Lemma 3.1 that every 6-traceable oriented graph of order $n$, where $6 \leq n \leq 12$ is traceable. However, the best that we've managed to do so far was to show that there does not exist a hypotraceable oriented graph of order less than 8 . To prove this, we need the following result, which is stated in (6) without proof.

Lemma 3.2 If $D$ is a hypotraceable digraph, then $D$ does not have a vertex with indegree 1 or outdegree 1 .

Proof: Let $D$ be a hypotraceable digraph, $x \in V(D)$ and suppose $y$ is the only out-neighbour of $x$. Then every hamiltonian path of $D-\{y\}$ must end in $x$, hence can be extended with $y$, which is a contradiction.

A strong digraph of order at least 2 cannot have a vertex of indegree 0 or outdegree 0 , so the following holds.

Corollary 3.3 If $D$ is a strong hypotraceable digraph, then $D$ has minimum indegree at least 2 and minimum outdegree at least 2.

We shall also use the following corollary of Lemma 2.2 . 
Corollary 3.4 Let $D$ be a hypotraceable oriented graph. If D contains a vertex $v$, such that $d^{+}(v)=2$ (or $d^{-}(v)=2$ ), then the out-neighbours (or in-neighbours) of $v$ are nonadjacent.

The following result is proved in (7).

Lemma 3.5 (Grötschel and Wakabayashi) Every nontrivial strong component of a hypotraceable oriented graph has order at least 5.

It is stated in (6) (without proof) that there does not exist a hypotraceable digraph of order less than 7. We now use the lemmas above to extend this bound in the case of oriented graphs.

Lemma 3.6 There does not exist a hypotraceable oriented graph of order less than 8.

Proof: Suppose $D$ is a hypotracable oriented graph of order $n$, with $3 \leq n \leq 7$.

Case 1. $D$ is strong: In this case Theorem 2.7 implies that $D$ has three independent vertices $\left\{x_{1}, x_{2}, x_{3}\right\}$ and it follows from Corollary 3.3 that $d^{+}\left(x_{1}\right) \geq 2$ and $d^{-}\left(x_{1}\right) \geq 2$. This is not possible if $n \leq 6$, so assume $n=7$. Then $d^{+}\left(x_{i}\right)=d^{-}\left(x_{i}\right)=2$ for $i=1,2,3$. Let $N^{+}\left(x_{1}\right)=\left\{a_{1}, a_{2}\right\}$ and $N^{-}\left(x_{1}\right)=$ $\left\{b_{1}, b_{2}\right\}$. By Corollary 3.4 $\left\{a_{1}, a_{2}\right\}$ and $\left\{b_{1}, b_{2}\right\}$ are independent sets. If $D-\left\{b_{1}, b_{2}\right\}$ has a hamiltonian path $Q$, then $Q$ starts at $x_{1}$ and ends at either $x_{2}$ or $x_{3}$, say $x_{3}$. But $d^{+}\left(x_{3}\right)=2$, so $x_{3}$ is adjacent to at least one of $b_{1}$ and $b_{2}$, say $b_{2}$. But then $b_{1} Q b_{2}$ is a hamiltonian path of $D$. This contradiction shows that $D-\left\{b_{1}, b_{2}\right\}$ has no hamiltonian path and hence $D-b_{1}$ has no hamiltonian path starting at $b_{2}$. Since $D$ is 6-traceable, $D-b_{1}$ has a hamiltonian path $P$. The initial vertex of $P$ cannot be $x_{1}$, otherwise $b_{1} P$ is a hamiltonian path of $D$. Without loss of generality, we assume that the initial vertex of $P$ is either $x_{2}$ or $a_{1}$.

Subcase 1.1 The initial vertex of $P$ is $x_{2}$ : In this case $b_{1} \notin N^{-}\left(x_{2}\right)$, otherwise $b_{1} P$ would be a hamiltonian path of $D$. Hence $b_{1} \in N^{+}\left(x_{2}\right)$. There are now two possibilities to consider for the second vertex of $P$.

Subcase 1.1.1 The second vertex of $P$ is $a_{1}$ : Then $N^{+}\left(x_{2}\right)=\left\{a_{1}, b_{1}\right\}$, so $N^{-}\left(x_{2}\right)=\left\{a_{2}, b_{2}\right\}$. If $a_{1} x_{3} \in A(D)$, then $x_{1} a_{2} x_{2} a_{1} x_{3}$ is a hamiltonian path in $D-\left\{b_{1}, b_{2}\right\}$, but we have shown $D-\left\{b_{1}, b_{2}\right\}$ is nontraceable, so $a_{1} \notin N^{-}\left(x_{3}\right)$. Also, $a_{2} \notin N^{-}\left(x_{3}\right)$, otherwise $b_{2} x_{2} b_{1} x_{1} a_{2} x_{3} a_{1}$ would be a hamiltonian path of $D$. Hence $N^{-}\left(x_{3}\right)=\left\{b_{1}, b_{2}\right\}$. But then $b_{2} x_{3} a_{2} x_{2} b_{1} x_{1} a_{1}$ is a hamiltonian path of $D$.

Subcase 1.1.2 The second vertex of $P$ is $b_{2}$ : Then $N^{+}\left(x_{2}\right)=\left\{b_{1}, b_{2}\right\}$, so $N^{-}\left(x_{2}\right)=\left\{a_{1}, a_{2}\right\}$. Then we may assume w.l.o.g. that $P$ is the path $x_{2} b_{2} x_{1} a_{1} x_{3} a_{2}$. But then $b_{2} x_{1} a_{1} x_{3} a_{2} x_{2} b_{1}$ is a hamiltonian path in $D$.

Subcase 1.2 The initial vertex of $P$ is $a_{1}$ : We have shown that $D-\left\{b_{1}, b_{2}\right\}$ has no hamiltonian path, so we may assume w.l.o.g. that $D-b_{1}$ has the hamiltonian path $a_{1} x_{2} b_{2} x_{1} a_{2} x_{3}$. Then $b_{1} \notin N^{+}\left(x_{3}\right)$, so $N^{+}\left(x_{3}\right)=\left\{a_{1}, b_{2}\right\}$. But then $b_{1} x_{3} a_{1} x_{2} b_{2} x_{1} a_{2}$ is a hamiltonian path in $D$.

Case 2. $D$ is not strong:

Subcase 2.1 $D$ has a nontrivial strong component $X$ that is nonhamiltonian: By Lemma 3.5, $|V(X)| \geq$ 5 , so $n=6$ or 7 . Since $D$ is $(n-1)$-traceable, Lemma 2.5 now implies that $|V(X)|=6$ and hence $n=7$. By symmetry, we may assume that $D_{1}$ has order one and $X=D_{2}$. Let $x$ be the vertex in $D_{1}$ and let $v_{1} v_{2} \ldots v_{6}$ be a path in $D_{2}$. Then $x, v_{6} \notin N^{-}\left(v_{1}\right)$, so it follows from Lemma 3.2 and Corollary 3.4 that $\left\{v_{3}, v_{5}\right\} \subseteq N^{-}\left(v_{1}\right)$. Similarly, $\left\{v_{2}, v_{4}\right\} \subseteq N^{+}\left(v_{6}\right)$. Hence each of the vertices $v_{1}, v_{4}$ and $v_{6}$ is an initial vertex of a hamiltonian path of $D_{2}$, so $x$ is not adjacent to $v_{1}, v_{4}$ or $v_{6}$. However, Lemma 3.2 implies that $d^{+}(x) \geq 2$ and, by Lemma $3.4 . N^{+}(x) \neq\left\{v_{2}, v_{3}\right\}$, so $v_{5} \in N^{+}(x)$. Then $v_{6} \notin N^{+}\left(v_{1}\right)$, otherwise 
$x v_{5} v_{1} v_{6} v_{2} v_{3} v_{4}$ is a hamiltonian path in $D$. Hence $N^{+}\left(v_{1}\right)=\left\{v_{2}, v_{4}\right\}$, which implies that $v_{2}$ and $v_{4}$ are nonadjacent vertices. But then $N^{+}\left(v_{4}\right)=\left\{v_{5}\right\}$, which contradicts Lemma 3.2.

Subcase 2.2 Every nontrivial strong component of $D$ is hamiltonian: In this case, if $D$ had only two strong components, $D$ would be traceable. Hence $D$ has at least three strong components. By Lemma 3.5. each nontrivial strong component of $D$ has order at least 5 . Thus the only possibility is that $n=7$ and $D$ has two trivial strong components and one of order 5 . The two trivial strong components cannot be consecutive, otherwise $D$ would be traceable. Hence $n\left(D_{1}\right)=1, n\left(D_{2}\right)=5$ and $n\left(D_{3}\right)=1$.

Let $x$ be the vertex in $D_{1}$, let $y$ be the vertex in $D_{3}$ and let $C=v_{1} v_{2} v_{3} v_{4} v_{5} v_{1}$ be a hamiltonian cycle of $D_{2}$. If $x$ has only one out-neighbour $v_{i}$ in $D_{2}$, then $D-v_{i}$ cannot be traceable, so $\left|N^{+}(x) \cap V\left(D_{2}\right)\right| \geq 2$. Similarly, $\left|N^{-}(y) \cap V\left(D_{2}\right)\right| \geq 2$. Since $D$ is nontraceable, no predecessor of a neighbour of $x$ on $C$ is a neighbour of $y$. Thus, at least one of $x$ and $y$ has at most two neighbours in $D_{2}$. By symmetry, we may assume that $x$ has only two out-neighbours in $D_{2}$, say $a$ and $b$. If $a b$ is an arc in $D$, then any hamiltonian path $x b \ldots v_{j} y$ of $D-a$ can be extended to a hamiltonian path $x a b \ldots v_{j}$ of $D$. Hence $a$ and $b$ are nonadjacent. We may assume, w.l.o.g. that $a=v_{2}$ and $b=v_{5}$. Then $v_{1}, v_{4} \notin N^{-}(y)$. But then $v_{1}$ has only four possible neighbours, namely $v_{2}, v_{3}, v_{4}, v_{5}$. Hence, by Lemma 3.2 and Corollary 3.4. $N^{+}\left(v_{1}\right)=\left\{v_{2}, v_{4}\right\}$ and $N^{-}\left(v_{1}\right)=\left\{v_{3}, v_{5}\right\}$. Now, if $v_{5}$ is adjacent to $y$, then $x v_{2} v_{3} v_{1} v_{4} v_{5} y$ is a hamiltonian path of $D$. Hence $v_{5}$ is not adjacent to $y$, so $N^{-}(y)=\left\{v_{2}, v_{3}\right\}$, which contradicts Corollary 3.4.

Lemmas 3.1 and 3.6 imply the following.

Corollary 3.7 If $2 \leq k \leq 7$, then every $k$-traceable oriented graph of order $n$ is traceable, where $k \leq n \leq 7$.

\section{Oriented graphs that are 6-traceable}

In this section we prove that the TC holds for $k=6$, i.e. that every 6-traceable oriented graph of order at least 11 is traceable. We first prove it for oriented graphs that are not strong.

Lemma 4.1 If $D$ is a 6-traceable oriented graph of order $n \geq 11$ that is not strong, then $D$ is traceable.

Proof: It follows from Lemma 2.6 that $D$ has a nontrivial strong component $X$ that is nonhamiltonian, and from Lemma 2.5 that $|V(X)|=n-1$.

By symmetry we may assume that $X=D_{2}$. Then $D_{1}$ has only one vertex $x$. Now $D_{2}$ is 5 -traceable and of order $n-1 \geq 10$. However, it is shown in (5) that the TC holds for $k=5$, so $D_{2}$ is traceable. Let $v_{1} v_{2} \ldots v_{n-1}$ be a hamiltonian path in $D_{2}$.

Let $v_{i}$ be a vertex in $D_{2}$ that is nonadjacent to $x$. If $d^{-}\left(v_{i}\right) \leq n-6$, then $D-N^{-}\left(v_{i}\right)$ has order at least 6 . But if $H$ is any subdigraph of $D-N^{-}\left(v_{i}\right)$ that has order 6 and contains both $v_{i}$ and $x$, then $H$ is nontraceable, since neither $x$ nor $v_{i}$ has any in-neighbour in $H$. This proves that every vertex in $D$ that is not a neighbour of $x$ has indegree at least $n-5$. In particular, $d^{-}\left(v_{1}\right) \geq n-5$. Hence it follows from Lemma 2.2 that $v_{3}, v_{n-2} \in N^{-}\left(v_{1}\right)$.

Since $X$ is strong, $v_{n-1}$ has an out-neighbour $v_{i}$ such that $2 \leq i \leq n-3$. If $v_{n-1} \in N^{+}(x)$, then $x v_{n-1} v_{i} \ldots v_{n-2} v_{1} \ldots v_{i-1}$ is a hamiltonian path of $D$. This contradiction shows that $v_{n-1}$ is not a neighbour of $x$ and hence $d^{-}\left(v_{n-1}\right) \geq n-5$.

By Lemma 2.1. $d^{+}(x) \geq n-5$, so $x$ has at least $n-5$ neighbours in the set $v_{2} \ldots,, v_{n-2}$. However, if $2 \leq i \leq n-2$ and $v_{i} \in N^{+}(x)$, then the predecessor $v_{i-1}$ is not in $N^{-}\left(v_{n-1}\right)$, otherwise $D$ has the 
hamiltonian path $x v_{i} \ldots v_{n-2} v_{1} \ldots v_{i-1} v_{n-1}$. Thus at least $n-5$ vertices in $D_{2}$ are not in-neighbours of $v_{n-1}$, which implies that $d^{-}\left(v_{n-1}\right) \leq 3$, contradicting that $d^{-}\left(v_{n-1}\right) \geq n-5 \geq 6$.

For the proof of the strong case, we also need the following result concerning 6-traceable oriented graphs that are not strong.

Lemma 4.2 If D is a 6-traceable oriented graph of order 8 that is not strong, then D is traceable.

Proof: Suppose $D$ is nontraceable. Then, since we have shown that there does not exist a hypotraceable oriented graph of order 7, Lemma 3.1 implies that $D$ itself is hypotraceable. We need to consider two cases.

Case 1. $D$ has a nontrivial strong component $X$ that is nonhamiltonian: It follows from Lemma 2.5 that $X$ has order 7. By symmetry we may assume that $D_{1}$ has order 1 and $D_{2}=X$. Let $x$ be the vertex in $D_{1}$ and let $v_{1} \ldots v_{7}$ be a path in $D_{2}$. It now follows exactly as in the proof of Lemma 4.1 that $v_{3}, v_{6} \in N^{-}\left(v_{1}\right)$ and also that $v_{1}$ and $v_{7}$ are not neighbours of $x$, and $d^{-}\left(v_{1}\right) \geq 3$ and $d^{-}\left(v_{7}\right) \geq 3$.

We now consider the possible neighbourhoods of $x$. By Lemma 2.1, $d^{+}(x) \geq 3$, and by Lemma 2.2, if $d^{+}(x)=3$, then $\left\langle N^{+}(x)\right\rangle$ is nontraceable. Moreover, if $v_{i} \in N^{+}(x)$, then $v_{i-1} \notin N^{-}\left(v_{7}\right)$.

Suppose $\left\{v_{2}, v_{6}\right\} \subseteq N^{+}(x)$. Then $N^{+}\left(v_{1}\right) \subseteq\left\{v_{2}, v_{4}, v_{5}\right\}$. But if $v_{4} \in N^{+}\left(v_{1}\right)$ then $x v_{2} v_{3} v_{1} v_{4} v_{5} v_{6} v_{7}$ is a hamiltonian path of $D$. Hence, by Lemma 3.2, $v_{5} \in N^{+}\left(v_{1}\right)$. But then $v_{4} \in N^{-}\left(v_{1}\right)$ and $x v_{2} v_{3} v_{4} v_{1} v_{5} v_{6} v_{7}$ is a hamiltonian path of $D$. This proves that $\left\{v_{2}, v_{6}\right\} \nsubseteq N^{+}(x)$, so we need to consider four cases.

Case $1.1\left\{v_{2}, v_{3}, v_{5}\right\} \subseteq N^{+}(x)$ : In this case $v_{1}, v_{2}, v_{4} \notin N^{-}\left(v_{7}\right)$. Hence $N^{-}\left(v_{7}\right)=\left\{v_{3}, v_{5}, v_{6}\right\}$. Since $v_{1} \notin N^{+}\left(v_{7}\right)$, this implies that $N^{+}\left(v_{7}\right)=\left\{v_{2}, v_{4}\right\}$. But then $x v_{3} v_{7} v_{4} v_{5} v_{6} v_{1} v_{2}$ is a hamiltonian path of $D$.

Case 1.2 $\left\{v_{2}, v_{4}, v_{5}\right\} \subseteq N^{+}(x)$ : In this case $N^{-}\left(v_{7}\right)=\left\{v_{2}, v_{5}, v_{6}\right\}$. But then $N^{+}\left(v_{7}\right)=\left\{v_{3}, v_{4}\right\}$, which contradicts Lemma 2.2 , since $D$ is hypotraceable.

Case $1.3\left\{v_{3}, v_{4}, v_{6}\right\} \subseteq N^{+}(x)$ : In this case $N^{-}\left(v_{7}\right)=\left\{v_{1}, v_{4}, v_{6}\right\}$. If either $v_{2}$ or $v_{3}$ is in $N^{+}\left(v_{7}\right)$, then $D$ would obviously be traceable. But then $d^{+}\left(v_{7}\right) \leq 1$, contradicting Lemma 3.2, since $D$ is hypotraceable.

Case $1.4\left\{v_{3}, v_{5}, v_{6}\right\} \subseteq N^{+}(x)$ : In this case $N^{-}\left(v_{7}\right)=\left\{v_{1}, v_{3}, v_{6}\right\}$. By Lemma 3.2, $d^{+}\left(v_{7}\right) \geq 2$. But by Corollary 3.4 $N^{+}\left(v_{7}\right) \neq\left\{v_{4}, v_{5}\right\}$. Hence $v_{2} \in N^{+}\left(v_{7}\right)$. Now if $v_{4} \in N^{-}\left(v_{1}\right)$, then $x v_{5} v_{6} v_{7} v_{2} v_{3} v_{4} v_{1}$ is a hamiltonian path in $D$. But we have shown that $d^{-}\left(v_{1}\right) \geq 3$, hence $v_{5} \in N^{-}\left(v_{1}\right)$. But then $x v_{6} v_{7} v_{2} v_{3} v_{4} v_{5} v_{1}$ is a hamiltonian path in $D$.

Case 2 Every strong component of $D$ is hamiltonian. In this case $D$ has at least three strong components, otherwise $D$ would be traceable. By Lemma 3.5, every nontrivial strong component of $D$ has order at least 5 . Hence the only possibility is that $N\left(D_{1}\right)=1, N\left(D_{2}\right)=6$ and $N\left(D_{3}\right)=1$.

Let $x$ and $y$ be the vertices in $D_{1}$ and $D_{3}$, respectively, and let $v_{0} v_{1} v_{2} v_{3} v_{4} v_{5} v_{0}$ be a hamiltonian cycle of $D_{2}$. If $x$ has only two neighbours in $D_{2}$, then removal of those two neighbours leaves a nontraceable subdigraph of $D$ that has order 6 . Hence $x$ has at least 3 neighbours in $D_{2}$ and, similarly, $y$ has at least three neighbours in $D_{2}$. But if $x$ is adjacent to $v_{i}$ and $j=(i-1) \bmod 6$, then $v_{j}$ cannot be adjacent to $y$. This implies that $\left|N^{+}(x) \cap V\left(D_{2}\right)\right|=3$ and $\left|N^{-}(y) \cap V\left(D_{2}\right)\right|=3$ and $N^{+}(x) \cap N^{-}(y) \neq \emptyset$. A similar argument to that used in Lemma 2.2 shows that both $\left\langle N^{+}(x) \cap V\left(D_{2}\right)\right\rangle$ and $\left\langle N^{-}(y) \cap V\left(D_{2}\right)\right\rangle$ are nontraceable.

If $\left|N^{+}(x) \cup N^{-}(y)\right| \leq 3$, then $D$ has a subdigraph of order 6 that contains at most one vertex in $N^{+}(x) \cap N^{-}(y)$. But such a subdigraph cannot be traceable. Hence $\left|N^{+}(x) \cup N^{-}(y)\right| \geq 4$. There are 
two possibilities to consider: $N^{+}(x)=\left\{v_{0}, v_{1}, v_{3}\right\}, N^{-}(y)=\left\{v_{1}, v_{3}, v_{4}\right\}$ and $N^{+}(x)=\left\{v_{0}, v_{1}, v_{4}\right\}$, $N^{-}(y)=\left\{v_{1}, v_{2}, v_{4}\right\}$. In the first case, since $D-\left\{v_{0}, v_{1}\right\}$ must be traceable, $D$ must contain the path $x v_{3} v_{5} v_{2} v_{4} y$. But then $D-\left\{v_{3}, v_{4}\right\}$ cannot be traceable. In the second case, $D-\left\{v_{1}, v_{2}\right\}$ is nontraceable, since $D_{2}-\left\{v_{1}, v_{2}\right\}$ does not have a path starting at $v_{0}$ and ending at $v_{4}$. Thus either case contradicts the 6-traceability of $D$.

We are now ready to prove our main theorem.

Theorem 4.3 Every 6-traceable oriented graph of order at least 11 is traceable.

Proof: Suppose, to the contrary that $D$ is a 6-traceable oriented graph of order $n$ that is nontraceable, for some $n \geq 11$. By Lemma 4.1 we may assume that $D$ is strong. Thus Theorem 2.9 implies that $n \leq 15$. By Theorem 2.7. $D$ has three independent vertices $x_{1}, x_{2}, x_{3}$. Let $A=N^{+}\left(x_{1}\right)$ and $B=$ $V(D) \backslash\left(A \cup\left\{x_{1}, x_{2}, x_{3}\right\}\right)$. Lemma 2.8 implies that $|A| \leq 6$ and $|B| \leq 6$. Hence, if $n=12$, then $|A| \geq 3$ and $|B| \geq 3$. If $n=11$, then it follows from Lemma 2.2 and the 6-traceability of $D$ that $|A| \neq 6$ and $|B| \neq 6$, so in this case $3 \leq|A| \leq 5$ and $3 \leq|B| \leq 5$.

If $|B|=3$, then put $S_{1}=B \cup\left\{x_{1}, x_{2}, x_{3}\right\}$ and $S_{2}=\left\{x_{1}\right\} \cup A$. If $B \geq 4$, then put $S_{1}=B \cup\left\{x_{1}, x_{2}\right\}$ and $S_{2}=\left\{x_{1}, x_{3}\right\} \cup A$. In either case, $6 \leq\left|S_{1}\right| \leq 8$ and $6 \leq\left|S_{2}\right| \leq 8, S_{1} \cup S_{2}=V(D)$ and $S_{1} \cap S_{2}=\left\{x_{1}\right\}$. Moreover, $x_{1}$ has no in-neighbours in $S_{2}$ and no out-neighbours in $S_{1}$, so neither $\left\langle S_{1}\right\rangle$ nor $\left\langle S_{2}\right\rangle$ is strong. Hence, it follows from the 6-traceability of $D$ and Theorem 3.6 and Lemma 4.2 that $S_{1}$ has a hamiltonian path ending in $x_{1}$ and $S_{2}$ has a hamiltonian path starting in $x_{1}$. But then $D$ has a hamiltonian path, contradicting our assumption.

Corollary 4.4 The OPPC(1) holds for $a \leq 5$.

Corollary 4.5 The OPPC(1) holds for oriented graphs of order at most 12.

\section{Acknowledgements}

The collaborative research for this paper was conducted while the first author was on a research visit to the P.J. Safarik University, which was funded by the National Scholarship Programme of the Slovak Republic. 


\section{References}

[1] J. Bang-Jensen and G. Gutin, Digraphs: Theory, Algorithms and Applications. Springer-Verlag, London, 2002.

[2] J. Bang-Jensen, M. Hegner Nielsen, A. Yeo, Longest path partitions in generalizations of tournaments. Discrete Math. 306 (2006) 1830-1839.

[3] C.C. Chen and P. Manalastas Jr., Every finite strongly connected digraph of stability 2 has a Hamiltonian path. Discrete Math. 44 (1983) 243-250.

[4] M. Frick, S. van Aardt, G. Dlamini, J. Dunbar and O. Oellermann, The directed path partition conjecture. Discuss. Math. Graph Theory. 25 (2005) 331-343.

[5] M. Frick, S.A. van Aardt, M.H. Nielsen, O. Oellermann and J.E. Dunbar, A Traceability conjecture for oriented graphs. Submitted.

[6] M. Grötschel, C. Thomassen and Y. Wakabayashi, Hypotraceable digraphs, J. Graph Theory 4 (1980) 377-381.

[7] M. Grötschel and Y. Wakabayashi, Constructions of hypotraceable digraphs, Mathematical Programming, Eds. R.W. Cottle, M.L. Kelmanson and B. Korte, Elsevier Science Publishers B.V. (North Holland), 1984.

[8] J.M. Laborde, C. Payan and N.H. Xuong, Independent sets and longest directed paths in digraphs, in: Graphs and other combinatorial topics (Prague,1982), 173-177 (Teubner-Texte Math. 59, 1983.)

[9] C. Thomassen, Hypohamiltonian graphs and digraphs. In Theory and Applications of graphs, Michigan 1976. Springer Lecture Notes, 642 (1978) 557 - 571.

[10] C.A. Whitehead, M. Frick and S.A. van Aardt, Significant differences between path partitions in directed and undirected graphs. To appear in Utilitas Math. 
